\title{
Estimation of Solid-liquid Interfacial Energy from Gibbs-Thomson Effect: A Molecular Dynamics Study
}

\author{
Ryo HASHIMOTO, Yasushi SHIBUTA* and Toshio SUZUKI \\ Department of Materials Engineering, The University of Tokyo, 7-3-1 Hongo, Bunkyo-ku, Tokyo, 113-8656 Japan. E-mail: \\ shibuta@material.t.u-tokyo.ac.jp
}

(Received on June 6, 2011; accepted on June 28, 2011)

\begin{abstract}
Solid-liquid interfacial energies of chromium and nickel are estimated from a Gibbs-Thomson relation. Molecular dynamics simulation shows that there is a critical temperature dividing shrinking or growing of a freestanding spherical crystal in the undercooled melt and the critical temperature is negatively correlated with inverse of crystal radius, which is regarded as the Gibbs-Thomson effect. The solid-liquid interfacial energies are then estimated from the proportional coefficient, to be $0.304 \mathrm{Jm}^{-2}$ for chromium and $0.256 \mathrm{Jm}^{-2}$ for nickel, respectively.
\end{abstract}

KEY WORDS: molecular dynamics simulation; solid-liquid interfacial energy; solidification; gibbs-thomson effect; nickel; chromium.

\section{Introduction}

Solid-liquid interfacial energy is one of the key factors determining microstructure of casting metal and alloy since it affects growth velocity of the dendrite and its tip radius during solidification. ${ }^{1)}$ In the earlier time, Turnbull ${ }^{2)}$ measured nucleation rate at temperature near maximum undercooling in the small droplets of various metals and calculated the solid-liquid interfacial energy of various metals on the basis of the homogeneous nucleation theory. The values estimated by Turnbull have been used for many years as a standard of the solid-liquid interfacial energy. Moreover, Gündüz and Hunt $^{3)}$ estimated the solid-liquid interfacial energy in the $\mathrm{Al}-\mathrm{Cu}, \mathrm{Al}-\mathrm{Si}$ and $\mathrm{Pb}-\mathrm{Sn}$ systems from the shape of grain boundary cusps of alloys obtained from annealing in a temperature gradient on the basis of GibbsThomson effect. Recently, Yasuda and coworkers ${ }^{4)}$ estimated the solid-liquid interfacial energy of the Sn-Bi alloy from the relation between tip radius and growth velocity of the dendrites for metallic alloys obtained from the in-situ observation of solidification process using synchrotron radiation X-ray. ${ }^{5)}$ Although these experimental works have contributed to accumulate a wealth of knowledge on the thermodynamic properties of the solid-liquid interface at high temperature, however, it is not straightforward to measure the solid-liquid interfacial energy at high temperature directly.

Therefore, theoretical and numerical studies have helped to interpret thermal and kinetic properties of the solid-liquid interface of metals and alloys. Especially, atomic simulation such as molecular dynamics (MD) method has contributed to understand the atomic behavior of the solid-liquid interface since it can trace atomic motion during solidification directly. For example, the kinetic coefficient of the solid-liquid interface has been estimated from MD simulations ${ }^{6-15}$ ) and these studies have given rise to a broad consensus about the anisotropy: the kinetic coefficient of the (100) plane is larger than those of the (110) and (111) plane for both $\mathrm{fcc}^{7)}$ and bec metals. ${ }^{8,9)}$ Regarding the solid-liquid interfacial energy, Broughton and Gilmer ${ }^{10)}$ and Davidchack and Laird $^{11)}$ have employed the cleaving technique to estimate the excess free energy at the solid-liquid interface. Moreover, the capillary fluctuation method $^{8,12,13)}$ has been employed for estimation of the solid-liquid interfacial energy from the capillary fluctuation spectrum of the roughness of the solid-liquid interface. Although these techniques have enabled the direct estimation of the solid-liquid interface from MD simulation, these are still some technical difficulty in these techniques. For example, many trials are needed to deal with the stable interface in the cleaving technique. Moreover, it is not easy to identify the solid and liquid atoms at the interface properly in the capillary fluctuation method to estimate interface stiffness, which relies on the criterion of one's choice. On the other hand, recent progress in the high performance computing has enabled MD simulation to treat a large system with more than hundreds of thousand atoms easily, which have provided a new technique for estimation of the solid-liquid interface using Gibbs-Thomson effect at curved interfaces. ${ }^{9,14,15)}$ For example, we have calculated the critical temperature dividing the growth and shrink of spherical crystals in the undercooling liquid with respect to particle size for iron system using Finnis-Sinclair (FS) potential ${ }^{16)}$ and revealed that the critical temperature was negatively correlated with the particle size, which was in line with the Gibbs-Thomson relation. ${ }^{9,15)}$ Then, the Gibbs-Thomson coefficient and the solid-liquid interface of iron were successfully estimated to be $1.9 \times 10^{-7}$ $\mathrm{mK}$ and $1.7 \times 10^{-1} \mathrm{~J} / \mathrm{m}^{2}$, respectively, from the GibbsThomson equation described below.

According to the classical nucleation theory, ${ }^{1)}$ a spherical solid crystal embedded in the undercooled melt causes a 

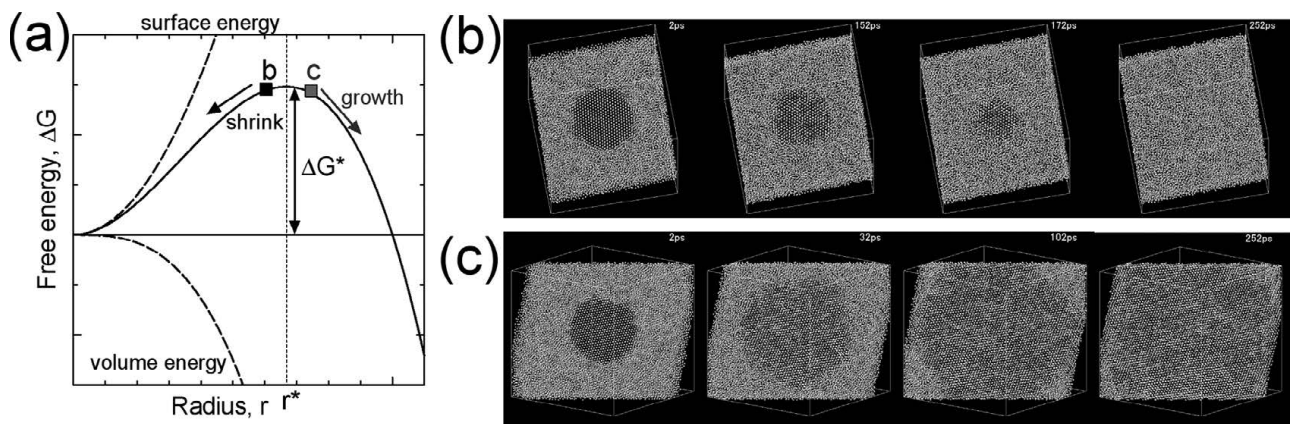

Fig. 1. (a) Schematic image of the free energy as a function of the radius of the spherical solid in the undercooled melt according to the classical nucleation theory. (b)(c) Snapshots of the cross-sectional view of the calculation cell containing free standing solid nanoparticles with radius of (b) 23.25 (5 488 atoms) and (c) $26.70 \AA$ (8 192 atoms) in the melt of $1950 \mathrm{~K}$. The write circles represent chromium atoms.

change in free energy $\Delta G$, expressed in terms of particle radius $r$ :

$$
\Delta G=4 \pi r^{2} \sigma_{\mathrm{SL}}-\frac{4 \pi r^{3}}{3} \frac{\Delta T \Delta H}{T_{M}},
$$

where $\sigma_{\mathrm{SL}}$ is the solid-liquid interfacial energy and $\Delta T$ is the degree of the undercooling, $\Delta H$ is the latent heat, and $T_{M}$ is the melting point. The change in free energy with respect to radius is convex and takes maximum value at the critical radius $r^{*}$ as illustrated in Fig. 1(a). The relationship between the critical radius and the degree of undercooling is derived from differentiation of the Eq. (1) to be zero as:

$$
\Delta T=\frac{2 \sigma_{\mathrm{SL}} T_{M}}{\Delta H} \frac{1}{r^{*}}=2 \Gamma \frac{1}{r^{*}}, \ldots
$$

where $\Gamma$ is the Gibbs-Thomson coefficient. Therefore, the solid-liquid interface can be derived when the Gibbs-Thomson coefficient, that is a correlation between the critical radius of the spherical particle in undercooled melt and the undercooling temperature, is estimated from the MD simulation directly. ${ }^{9,14,15)}$ In this study, this methodology is employed to calculate the solid-liquid interfacial energy of both the bcc (chromium) and fec (nickel) metals using two different interatomic potential and the validity of the methodology is then discussed.

\section{Simulation Methodology}

The $\mathrm{FS}^{16)}$ and a long-range FS potential called SuttonChen $(\mathrm{SC})^{17)}$ potentials were applied to describe the interaction between chromium and nickel atoms, respectively. The FS potential is one of the established potential for metals since it can reproduce the material properties despite its simple form. We have employed these potentials consistently in a series of calculations of the depression of the melting point of freestanding metal nanoparticles with respect to particle radius for both the $\mathrm{bcc}^{18-20)}$ and fcc metals ${ }^{21)}$ and confirmed a reproducibility of the linearity between the depression of the melting point and the particle radius properly. The total energy of the FS potential, $E$, is expressed in following equations:

$$
\begin{array}{r}
E=\frac{1}{2} \sum_{i} \sum_{j} V_{i j}\left(r_{i j}\right)-A \sum_{i} \sqrt{\rho_{i}} \ldots \ldots . \\
V_{i j}= \begin{cases}\left(r_{i j}-c\right)^{2}\left(c_{0}+c_{1} r_{i j}+c_{2} r_{i j}^{2}\right) & \left(r_{i j} \leq c\right) \\
0 & \left(r_{i j}>c\right)\end{cases}
\end{array}
$$

Table 1. Parameters of Finnis-Sinclair potential for chromium. ${ }^{16)}$

\begin{tabular}{ccccccc}
\hline$d(\AA)$ & $A(\mathrm{eV})$ & $\beta$ & $C(\AA)$ & $C_{0}$ & $C_{1}$ & $C_{2}$ \\
\hline 3.915720 & 1.453418 & 1.8 & 2.90 & 29.1429813 & -23.3975027 & 4.7578297 \\
\hline
\end{tabular}

Table 2. Parameters of Sutton-Chen potential for nickel. ${ }^{17)}$

\begin{tabular}{ccccc}
\hline$a(\AA)$ & $\varepsilon(\mathrm{eV})$ & $c$ & $m$ & $n$ \\
\hline 3.52 & $1.5713 \times 10^{-2}$ & 39.755 & 6 & 9 \\
\hline
\end{tabular}

$$
\begin{aligned}
& \rho_{i}=\sum_{j \neq i} \phi\left(r_{i j}\right) \\
& \phi\left(r_{i j}\right)= \begin{cases}\left(r_{i j}-d\right)^{2}+\beta \frac{\left(r_{i j}-d\right)^{3}}{d} & \left(r_{i j} \leq d\right), \\
0 & \left(r_{i j}>d\right)\end{cases}
\end{aligned}
$$

where $V_{i j}$ is the repulsive term, $A$ is the binding energy, $r_{i j}$ is the bond length between atoms $i$ and $j, \rho$ is the total electronic charge density at the site of atom $i$, which is constructed by the rigid superposition of atomic charge densities $\phi$. The parameters are taken from the original work by Finnis and Sinclair ${ }^{16)}$ (Table 1). Meanwhile, the total energy of the SC potential, $E$, is expressed in the following equation:

$$
E=\varepsilon\left[\frac{1}{2} \sum_{i} \sum_{j} V\left(r_{i j}\right)-c \sum_{i} \sqrt{\rho_{i}}\right],
$$

where $\varepsilon$ is the parameter with the dimension of energy and $c$ is the positive dimension parameter. The repulsive term and total electronic charge density in the Eq. (7) are expressed using following polynomial equations:

$$
\begin{aligned}
& V\left(r_{i j}\right)=\left(\frac{a}{r_{i j}}\right)^{n} . \\
& \rho_{i}=\sum_{j \neq i}\left(\frac{a}{r_{i j}}\right)^{m},
\end{aligned}
$$

where $a$ is the lattice constant and the parameter $n$ and $m$ are positive integers with $n>m$. The parameters recomputed by Rafii-Tabar and Sutton ${ }^{22)}$ are employed (Table 2), which are fitted for the use of a cut-off radius of twice the length of lattice constant. As a simulation methodology of the MD 
calculation, a leapfrog method was used to integrate a classical equation of motion with time step of $5.0 \mathrm{fs}$. The Berendsen thermostat ${ }^{23)}$ was applied to control the temperature for every step and the Andersen method ${ }^{24)}$ was applied for controlling pressure in each direction independently.

\section{Results and Discussion}

In order to estimate the solid-liquid interfacial energy from the Gibbs-Thomson effect, the growing and shrinking behavior of spherical crystals embedded in the undercooled liquid is calculated in the following manner. ${ }^{9,15)}$ The initial configuration was prepared by inserting a solid nanoparticle in the melt. The solid nanoparticles were obtained by cooling freestanding liquid droplets from 3000 to $0 \mathrm{~K}$ for chromium $^{19)}$ and from 1500 to $0 \mathrm{~K}$ for nickel ${ }^{21)}$ at a rate of $1 \mathrm{~K} / 5$ ps. The number of atoms in the nanoparticle varied from 1024 to $16000\left(2 n^{3}: n=8-20\right)$ for chromium and from 2048 and $32000\left(4 n^{3}: n=8-20\right)$ for nickel, respectively. The liquid cells were obtained by heating bcc crystal for chromium and fcc crystal for nickel at $3500 \mathrm{~K}$. The number of atoms in the cell filled with the melt varied from 8192 to $128000\left(16 n^{3}: n=8-20\right)$ for chromium and 16384 to $256000\left(32 n^{3}: n=8-20\right)$ for nickel, respectively. For each pair, the solid nanoparticle were inserted into the liquid cell, while omitting all liquid atoms located within $2.5 \AA$ from a solid atom to avoid the unexpected proximity between liq-
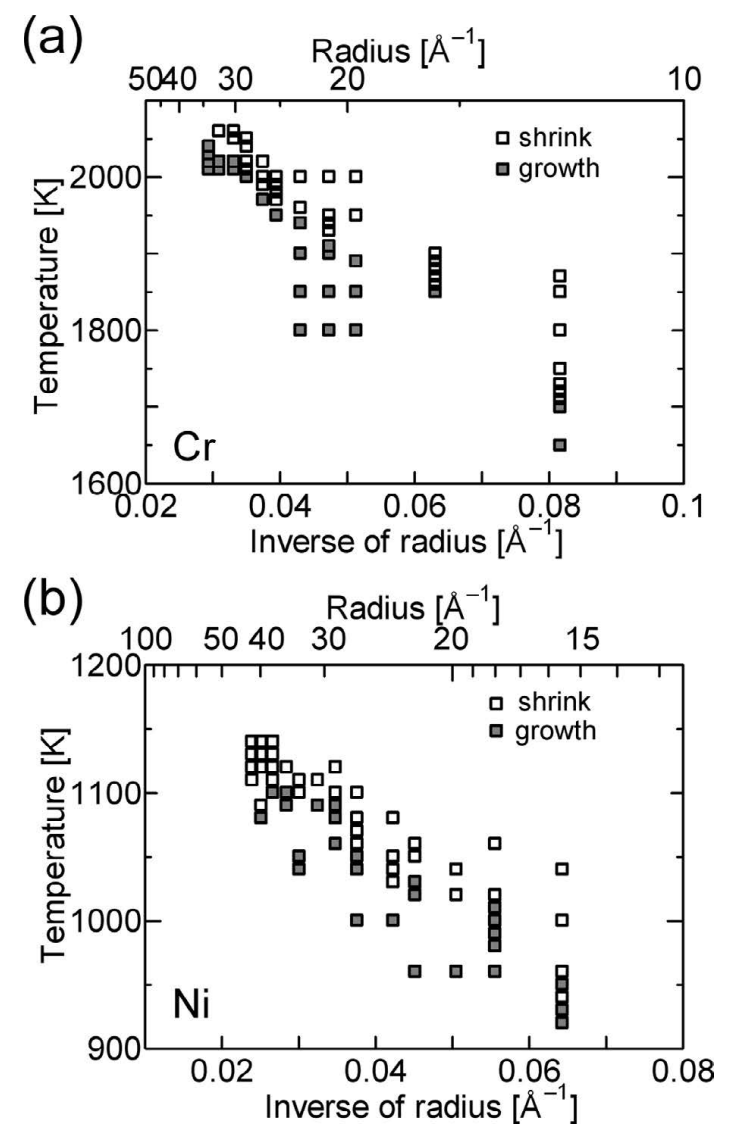

Fig. 2. Matrix showing the direction of growth or shrink as a function of temperature and the inverse of particle radius of the initial solid nanoparticle for (a) chromium and (b) nickel. White and Black square represent the melting and solidification, respectively. uid and solid atoms at the interface. The system was then quenched at $10 \mathrm{~K}$ for 2 ps to fill the gap between solid nanoparticle and liquid melt. The obtained structure was employed as the initial configuration of the main calculation.

Each initial configuration was then relaxed under zero pressure at various temperatures below the melting point. The relaxation was examined with a $10 \mathrm{~K}$ interval around the critical temperature. Figures 1(b) and 1(c) show the snapshots of cross-sectional view of the cell with solid nanoparticles consisting of $5488(r=23.25 \AA)$ and 8192 atoms $(r=26.70 \AA)$ during relaxation at $1950 \mathrm{~K}$. It was observed that the smaller nanoparticles shrunk and melted in the melt, whereas bigger one grew as a result of solidification. This behavior agrees with trend in the free energy with respect to particle size as illustrated in Fig. 1(a). Therefore, it is expected that there is a critical radius dividing growth and shrink of the nanoparticle between 23.25 and $26.70 \AA$ at this temperature. Figure 2 is a matrix showing the direction of growth or shrink as a function of temperature and the inverse of initial particle radius for chromium and nickel. All the spherical nanoparticle did not remain but grew or shrank, since the free energy of the system with respect to particle radius is convex and takes local maximum at the critical radius as illustrated in Fig. 1(a). The critical radius should exist between the largest radius for shrink and the smallest one for growth and then it can be defined as the intermediate distance between them. On the other hand, there is a critical temperature dividing shrink and growth of the solid particle in the melt as the point of view of constant radius. Therefore, the critical temperature can be defined between the lowest melting and the largest solidification temperature in a similar way.

The critical temperatures were then summarized in the form of the undercooling temperature from the bulk melting point with respect to the inverse of initial particle radius in Fig. 3. Here, the extrapolation temperature of the inverse of particle radius to zero ${ }^{19,21)}$ was employed as the bulk melting point. The depression of the critical temperature from the bulk melting point was proportional to the inverse of the critical radius for both the case of the chromium and nickel,

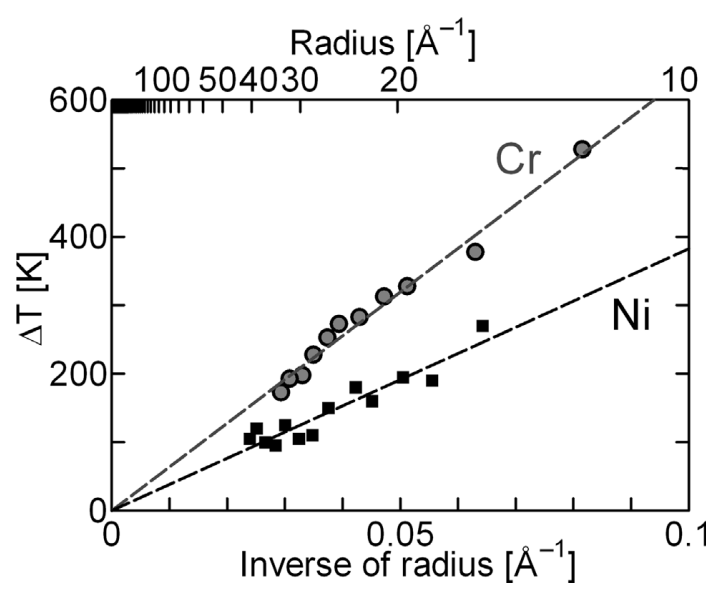

Fig. 3. Depression of the critical temperature from the bulk melting point as a function of the inverse of the initial particle radius. Gray dots and black squares represent data for chromium and nickel, respectively. 
which agrees with the Gibbs-Thomson relation described above. However, depression of the critical temperature for nickel was not monotonic but had some fluctuation with decreasing particle radius, whereas that for chromium was monotonic with respect to the inverse of particle radius. This difference was also observed in the trend of depression of the melting point of freestanding nanoparticle with respect to particle radius between bcc and fcc metals, ${ }^{19,21)}$ which was caused by the difference of the structure in the nanoparticles: single crystal for the bcc nanoparticles and polycrystal for fcc nanoparticles. ${ }^{21)}$ However, there was a negative correlation between depression of the critical temperature and critical radius for both metals as a whole. Therefore, the Gibbs-Thomson coefficient and solid-liquid interfacial energy can be estimated from the slope of the fitting line in Fig. 3. The Gibbs-Thomson coefficients for chromium and nickel were estimated to be $3.19 \times 10^{-7}$ and $1.91 \times 10^{-7} \mathrm{mK}$, respectively. The solid-liquid interfacial energy were then calculated by substituting the latent heat $\left(0.16 \mathrm{eV} /\right.$ atom for chromium $\left.{ }^{19}\right)$ and $0.11 \mathrm{eV} /$ atom for nickel $^{21)}$ ) and melting point of the bulk system into the Gibbs-Thomson coefficient, to be $3.04 \times 10^{-1} \mathrm{~J} / \mathrm{m}^{2}$ for chromium and $2.56 \times 10^{-1} \mathrm{~J} / \mathrm{m}^{2}$ for nickel, respectively. These results are summarized in Table 3. Several reference values from literatures estimated using different interatomic potentials and from Turnbull's experiments are listed for comparison. Properties of the iron are also listed for reference. It was confirmed that the solid-liquid interfacial energy of nickel estimated in this study is relatively close to the experimental values reported by Turnbul1 ${ }^{2)}$ and other values estimated from other numerical works. However, it is difficult to find the correlation among the values estimated from var-

Table 3. Melting point, $T_{M}$, latent heat, $\Delta H$, Gibbs-Thomson coefficient, $\Gamma$ and solid-liquid interfacial energy, $\sigma_{\mathrm{SL}}$ estimated using Finnis-Sinclair potential for chromium and SuttonChen potential for nickel. Those from the Turnbull's experiment and the calculations using other interatomic potentials are also listed for comparison. In addition, those properties of the iron are plotted for reference.

\begin{tabular}{lcccc}
\hline \multicolumn{1}{c}{$\begin{array}{c}\text { Element } \\
\text { (potential) }\end{array}$} & $\begin{array}{c}T_{M} \\
(\mathrm{~K})\end{array}$ & $\begin{array}{c}\Delta H \\
(\mathrm{eV} / \text { atom })\end{array}$ & $\begin{array}{c}\Gamma \\
(\mathrm{mK})\end{array}$ & $\begin{array}{c}\sigma_{\mathrm{SL}} \\
\left(\mathrm{J} / \mathrm{m}^{2}\right)\end{array}$ \\
\hline $\mathrm{Cr}(\mathrm{FS})$ & 2232 & $0.16^{19)}$ & $3.19 \times 10^{-7}$ & $3.04 \times 10^{-1}$ \\
$\mathrm{Cr}($ Experiment) & 2180 & 0.22 & - & - \\
$\mathrm{Ni}(\mathrm{SC})$ & 1205 & $0.11^{21)}$ & $1.91 \times 10^{-7}$ & $2.56 \times 10^{-1}$ \\
$\mathrm{Ni}(\mathrm{SMF})^{\mathrm{a}}$ & 1820 & 0.187 & - & $3.10 \times 10^{-1}$ \\
$\mathrm{Ni}($ Experiment) & 1728 & 0.181 & - & $2.55 \times 10^{-1}$ \\
$\mathrm{Fe}(\mathrm{FS})^{\mathrm{c}}$ & $2400 \pm 10^{25)}$ & 0.22 & $1.9 \times 10^{-7}$ & $1.70 \times 10^{-1}$ \\
$\mathrm{Fe}(\mathrm{ABCH})^{\mathrm{d}}$ & $2358 \pm 4$ & 0.218 & - & $2.06 \times 10^{-1}$ \\
$\mathrm{Fe}($ Experiment) & 1811 & 0.143 & $1.9 \times 10^{-71}$ & $2.04 \times 10^{-1}$ \\
\hline
\end{tabular}

a Asta et al. ${ }^{26,27)}$

b Turnbull ${ }^{2)}$

c Watanabe et al. ${ }^{9)}$

d Sun et al. ${ }^{8)}$ ious interatomic potentials in this stage. We note that we have to be careful to interpret these results from a quantitative point of view since accuracy of the estimated values strongly depends on that of the interatomic potential.

\section{Conclusions}

In this study, the solid-liquid interfacial energies of chromium and nickel were estimated from a Gibbs-Thomson relation, which was found in the MD simulation on the growth and shrink of the solid particle in the undercooled melt. The validity of this methodology was then discussed by comparing the solid-liquid liquid interfacial energy of nickel obtained in this study with those from literatures estimated using different interatomic potentials and from Turnbull's experiments. It is significant in this study that we have proposed and validated a new methodology that can estimate the solid-liquid interfacial energy from Gibbs-Thomson relation, since the estimation of the solid-liquid interfacial energy has not been established. This methodology will enable the systematic survey of the solid-liquid interfacial energy for various metals including high melting point metals whose properties are difficult to be measured directly. In addition, further improvement of the interatomic potentials will raise the precision of the estimated value.

\section{Acknowledgements}

Part of this work was financially supported by the 20th ISIJ Research Promotion Grant from The Iron and Steel Institute of Japan.

\section{REFERENCES}

1) W. Kurz and D. J. Fisher: Fundamental of Solidification 4th revised edition, Trans Tech Publication, Aedermannsdorf, (1998), 21.

2) D. Turnbull: J. Appl. Phys., 21 (1950), 1022.

3) M. Gündüz and J. D. Hunt: Acta Metall., 33 (1985), 1651.

4) H. Yasuda, T. Nagira, M. Yoshiya, A. Sugiyama, K. Uesugi and K. Umentani: J. Jpn. Assoc. Cryst. Growth, 37 (2010), 82.

5) H. Yasuda, I. Ohnaka, K. Kawasaki, A. Sugiyama, T. Ohmichi, J. Iwane and K. Umetani: J. Cryst. Growth, 262 (2004), 645.

6) J. Q. Broughton, G. H. Gilmer and K. A. Jackson: Phys. Rev. Lett., 49 (1982), 1496

7) J. J. Hoyt, B. Sadigh, M. Asta and S. M. Foiles: Acta Mater., 47 (1999), 3181.

8) D. Y. Sun, M. Asta and J. J. Hoyt: Phys. Rev. B, 69 (2004), 174103.

9) Y. Watanabe, Y. Shibuta and T. Suzuki: ISIJ Int., 50 (2010), 1158.

10) J. Q. Broughton and G. H. Gilmer: J. Chem. Phys., 84 (1986), 5759.

11) R. L. Davidchack and B. B. Laird: J. Chem. Phys., 118 (2003), 7651.

12) J. J. Hoyt, M. Asta and A. Karma: Phys. Rev. Lett., 86 (2001), 5530.

13) J. R. Morris and X. Song: J. Chem. Phys., 119 (2003), 3920.

14) X.-M. Bai and M. Li: J. Chem. Phys., 124 (2006), 124707.

15) Y. Shibuta, Y. Watanabe and T. Suzuki: Chem. Phys. Lett., 475 (2009), 264.

16) M. W. Finnis, J. E. Sinclair: Philos. Mag. A, 50 (1984), 45

17) A. P. Sutton and J. Chen: Philos. Mag. Lett., 61 (1990), 139.

18) Y. Shibuta and T. Suzuki: Chem. Phys. Lett., 445 (2007), 265.

19) Y. Shibuta and T. Suzuki: J. Chem. Phys., 129 (2008), 144102

20) Y. Shibuta and T. Suzuki: Chem. Phys. Lett., 502 (2011), 82.

21) Y. Shibuta and T. Suzuki: Chem. Phys. Lett., 498 (2010), 323

22) H. Rafii-Tabar and A. P. Sutton: Philos. Mag. Lett., 63 (1991), 217.

23) H. J. C. Berendsen, J. P. M. Postma, W. F. van Gunsteren, A. DiNola and J. R. Haak: J. Chem. Phys., 81 (1984), 3684.

24) H. C. Andersen: J. Chem. Phys., 72 (1980), 2384.

25) Y. Shibuta, S. Takamoto and T. Suzuki: ISIJ Int., 48 (2008), 1582.

26) M. Asta, J. J. Hoyt and A. Karma: Phys. Rev. B, 66 (2002), 100101.

27) J. J. Hoyt, M. Asta and A. Karma: Mater. Sci. Eng. R, 41 (2003), 121. 